\title{
Spherically-symmetric model stellar atmospheres and limb darkening
}

\section{Limb-darkening laws, gravity-darkening coefficients and angular diameter corrections for red giant stars ${ }^{\star}$}

\author{
H. R. Neilson ${ }^{1}$ and J. B. Lester ${ }^{2,3}$ \\ ${ }^{1}$ Department of Physics \& Astronomy, East Tennessee State University, Box 70652, Johnson City, TN 37614, USA \\ e-mail: neilsonh@etsu.edu \\ 2 Department of Chemical and Physical Sciences, University of Toronto Mississauga, ON L5L 1C6, Canada \\ 3 Department of Astronomy \& Astrophysics, University of Toronto, ON M55, 3H4, Canada \\ e-mail: lester@astro.utoronto.ca \\ Received 18 March 2013 / Accepted 30 April 2013
}

ABSTRACT

\begin{abstract}
Model stellar atmospheres are fundamental tools for understanding stellar observations from interferometry, microlensing, eclipsing binaries and planetary transits. However, the calculations also include assumptions, such as the geometry of the model. We use intensity profiles computed for both plane-parallel and spherically symmetric model atmospheres to determine fitting coefficients in the BVRIHK, CoRot and Kepler wavebands for limb darkening using several different fitting laws, for gravity-darkening and for interferometric angular diameter corrections. Comparing predicted variables for each geometry, we find that the spherically symmetric model geometry leads to different predictions for surface gravities $\log g<3$. In particular, the most commonly used limb-darkening laws produce poor fits to the intensity profiles of spherically symmetric model atmospheres, which indicates the need for more sophisticated laws. Angular diameter corrections for spherically symmetric models range from 0.67 to 1 , compared to the much smaller range from 0.95 to 1 for plane-parallel models.
\end{abstract}

Key words. stars: atmospheres - stars: late-type - binaries: eclipsing - stars: evolution - techniques: interferometric

\section{Introduction}

Stellar limb darkening is an important tool for interpreting interferometric, microlensing and eclipsing binary observations of red giant and supergiant stars. It also provides critical information about the temperature structure of a stellar atmosphere (Schwarzschild 1906) as well as a measure of the radial extension of an atmosphere (Neilson \& Lester 2012).

Interferometric observations measure the angular diameter of a star as well as the intensity variation across the stellar surface. Some of the first interferometric observations measured only uniform-disk angular diameters, that is the angular diameter for a star assumed to have a constant surface brightness (Hanbury Brown et al. 1974). Wittkowski et al. (2004) presented $K$-band interferometric observations of the M3 giant $\psi$ Phoenicis with measurements of the first and second lobes of the visibility curve, which constrain limb darkening. Unfortunately, these observations were not precise enough to distinguish between different model stellar atmospheres. Advances in interferometric observations have allowed for observations of convective cells in Betelgeuse (Haubois et al. 2009) and measurements of gravity darkening in Altair (van Belle et al. 2001). In terms of model stellar atmospheres, Aufdenberg et al. (2005) constrained threedimensional models using observations of Procyon.

* Tables $2-17$, and the model intensity profiles are only available at the CDS via anonymous ftp to

cdsarc.u-strasbg.fr (130.79.128.5) or via

http://cdsarc.u-strasbg.fr/viz-bin/qcat?J/A+A/554/A98
Microlensing observations, like interferometry, also probe stellar limb darkening, but unlike interferometry, which targets specific nearby stars, microlensing observations are random. An et al. (2002) and Fields et al. (2003) constrained non-linear limb-darkening relations from microlensing observations of a $\mathrm{K} 3$ giant and compared them to model stellar atmospheres. They found significant disagreement between the observed and predicted limb-darkening relation. More recently, however, microlensing observations have only constrained linear limb-darkening relations for red giant stars (Fouqué et al. 2010; Zub et al. 2011).

Eclipsing binaries and planetary transits provide yet another avenue for measuring stellar limb darkening. In terms of red giant stars, there are a number of known eclipsing binary systems, specifically the $\zeta$ Aurigae systems that have a K4-5 red giant primary and a main-sequence B-type companion. Eaton et al. (2008) fit the orbits for several of these systems assuming a simple linear limb-darkening law. There is also the potential of observing planets transiting red giant stars, which would provide powerful constraints of theories of planetary evolution. Currently, extrasolar planets have been observed orbiting dwarf and subgiant stars (Howell et al. 2012), but not giant stars; future missions such as PLATO may remedy this (Catala et al. 2010).

These three types of observations are ideal tools for probing stellar atmospheres and constraining the physics employed in numerical models. Likewise, predictions from model stellar atmospheres help constrain these types of observations. Recently, Sing (2010), Howarth (2011a) and Claret \& Bloemen (2011) presented limb-darkening laws fit to plane-parallel model stellar 
atmosphere intensity profiles. Even more recently, Claret et al. (2012, 2013) fit limb-darkening laws to spherically-symmetric PHOENIX model stellar atmospheres of cool brown dwarf stars. In this work, we study how the assumed geometry of the model stellar atmosphere, plane parallel versus spherically symmetric, affects predictions of stellar limb darkening, gravity darkening and interferometric angular diameter corrections. We examine model atmospheres spanning the effective temperature and gravity range consistent with yellow and red giant and supergiant stars. Tables of limb-darkening and gravity-darkening coefficients, as well as new angular diameter corrections are presented as more physically based tools for understanding these bright stars.

In Sect. 2 we describe the stellar atmosphere code used in this work, as well as the model atmosphere grids computed for both plane-parallel and spherically symmetric geometries. In Sect. 3 limb-darkening coefficients are presented for several commonly used limb-darkening relations. We compute gravitydarkening coefficients in Sect. 4 and angular diameter corrections in Sect. 5. Computations in these three sections provide insight into how intensity profiles depend on the assumed model geometry that can be directly compared to observations.

\section{Model stellar atmospheres}

Model stellar atmospheres form a key foundation of our understanding of stars, arguably a great success of computational astrophysics. However, the early success of model atmosphere codes transformed them into standard tools, and only in the past decade have these codes moved beyond simple plane-parallel, local-thermodynamic-equilibrium (LTE) models to full threedimensional, statistical-equilibrium codes that can model nonLTE physics as well as stellar convection. Unfortunately, computing power is still limited for calculating large-scale model atmosphere grids varying stellar gravity, effective temperature, stellar mass and composition.

A step toward more realistic geometry is achieved by shifting from one-dimensional plane-parallel model stellar atmosphere codes to one-dimensional spherically symmetric codes, which can be used to compute large grids of models atmospheres that include physics that is more appropriate to stars where the depth of the stellar photosphere is a significant fraction of the stellar radius, such as evolved giant and supergiant stars and pre-main sequence stars. One such code for modeling atmospheres assuming spherically symmetric geometry is the SATLAS code (Lester $\&$ Neilson 2008). This code is based on the ATLAS code developed by Kurucz (1979), and continues its assumption of local thermodynamic and hydrostatic equilibrium. However, the radiative transfer is computed assuming spherical geometry using the Rybicki (1971) version of the Feautrier (1964) ray-tracing method, while radiative and convecting equilibrium is enforced using an updated version of the Avrett \& Krook (1963) temperature correction method. Models computed using this code have been compared to spherically-symmetric PHOENIX and MARCS models (Hauschildt et al. 1999; Gustafsson et al. 2008) and shown to produce similar results (Lester \& Neilson 2008; Neilson \& Lester 2008).

In this work we use the grid of spherical model atmospheres from Neilson \& Lester (2011), extended in mass up to $M=$ $20 M_{\odot}$. The grid assumes solar composition and spans the gravities from $\log g=-1$ to $\log g=3$ in steps of 0.25 , effective temperatures from $T_{\text {eff }}=3000$ to $8000 \mathrm{~K}$ and masses from $M=2.5$ to $20 M_{\odot}$ in steps of $2.5 M_{\odot}$ and includes models with masses $M=0.5$ and $1 M_{\odot}$. Surface intensities are computed for each model at 1000 equally spaced values of $\mu=\cos \theta$, where $\theta$ is the angle between the vertical direction and the direction toward a distant observer. Limb-darkening profiles are computed for Johnson-Cousins BVRIHK-wavebands (Johnson \& Morgan 1953; Bessell 2005) along with the CoRot (Auvergne et al. 2009) and Kepler (Koch et al. 2004) wavebands. Angular diameter corrections for interferometric observations, gravity-darkening coefficients and various limb-darkening relations are computed using these wavelength-integrated intensity profiles.

\section{Limb-darkening laws}

An understanding of stellar limb darkening is required to model the properties of interferometric, eclipsing binary-star, microlensing, and planetary-transit observations. As these observations become more precise and more accurate, models of stellar limb darkening must also improve. Limb darkening is typically treated as a simple parametrization as a function of $\theta$ (e.g. Fouqué et al. 2010; Croll et al. 2011), which makes fitting the stellar intensity profile much simpler and reduces the number of free parameters. The most common parametrizations are linear and quadratic relations (Al-Naimiy 1978; van Hamme 1993; Diaz-Cordoves et al. 1995), but other suggested relations include a four-parameter relation (Claret 2000a), a square-root relation (Wade \& Rucinski 1985) as well as exponential and logarithmic relations (Claret 2000a; Claret \& Hauschildt 2003).

\subsection{Best-fit limb-darkening laws}

We fit the following limb-darkening relations to the grids of plane-parallel and spherically symmetric model stellar atmospheres:

$\begin{array}{lr}\frac{I(\mu)}{I(\mu=1)}=1-u(1-\mu) & \text { Linear, } \\ \frac{I(\mu)}{I(\mu=1)}=1-a(1-\mu)-b(1-\mu)^{2} & \text { Quadratic, }\end{array}$

$\frac{I(\mu)}{I(\mu=1)}=1-c(1-\mu)-d(1-\sqrt{\mu}) \quad$ Square-Root,

$\frac{I(\mu)}{I(\mu=1)}=1-\sum_{j=1}^{4} f_{j}\left(1-\mu^{j / 2}\right) \quad$ 4-Parameter,

$\frac{I(\mu)}{I(\mu=1)}=1-g(1-\mu)-h \frac{1}{1-e^{\mu}} \quad$ Exponential,

$\frac{I(\mu)}{I(\mu=1)}=1-m(1-\mu)-n \mu \ln \mu \quad$ Logarithmic.

We derive the best-fit coefficients for each of the limb-darkening laws using a general least-squares algorithm. This was done using the computed surface intensities for the BVRIHK- and CoRot- and Kepler-wavebands. Figure 1 shows the Kepler-band intensity profile and corresponding best-fit limb-darkening laws for both spherical and plane-parallel model atmospheres with the properties $T_{\text {eff }}=5000 \mathrm{~K}, \log g=2$ and $M=10 M_{\odot}$ (mass is defined for the spherical model only). The chosen limb-darkening laws all fit the plane-parallel model intensity profiles well. This is not surprising because plane-parallel model atmosphere intensity profiles do not deviate significantly from being linear, and a linear term is included in all of the chosen limb-darkening laws. However, spherically-symmetric model stellar atmospheres have intensity profiles that are significantly non-linear, and the bestfit limb-darkening relations for these intensity profiles match 
H. R. Neilson and J. B. Lester: Limb darkening in red giant stars
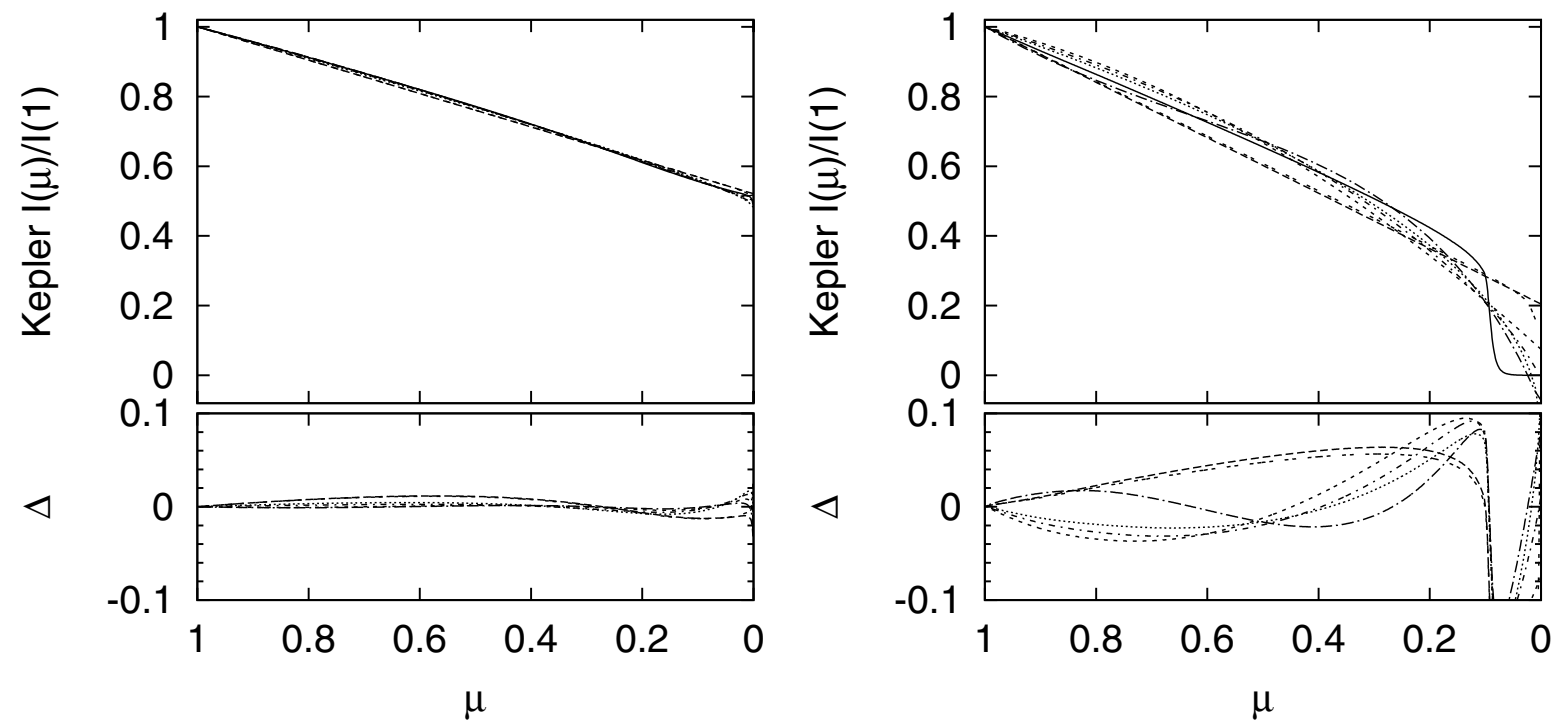

Fig. 1. Kepler-band model intensity profiles (black-solid) predicted for both plane-parallel (left) and spherically symmetric (right) model stellar atmospheres with $T_{\text {eff }}=5000 \mathrm{~K}, \log g=2$ and $M=10 M_{\odot}$. Along with the intensity profiles, best-fit linear (green-dashed), quadratic (orangeshort-dashed), square-root (blue-dotted), four-parameter (violet-long-dash-dotted), logarithmic (brown-short-dash-dotted), and exponential (greydouble-dash) limb-darkening relations are plotted. Bottom panels show the difference, $\Delta \equiv I_{\text {model }}-I_{\text {law }}$, between model intensities and best-fit limb-darkening laws.

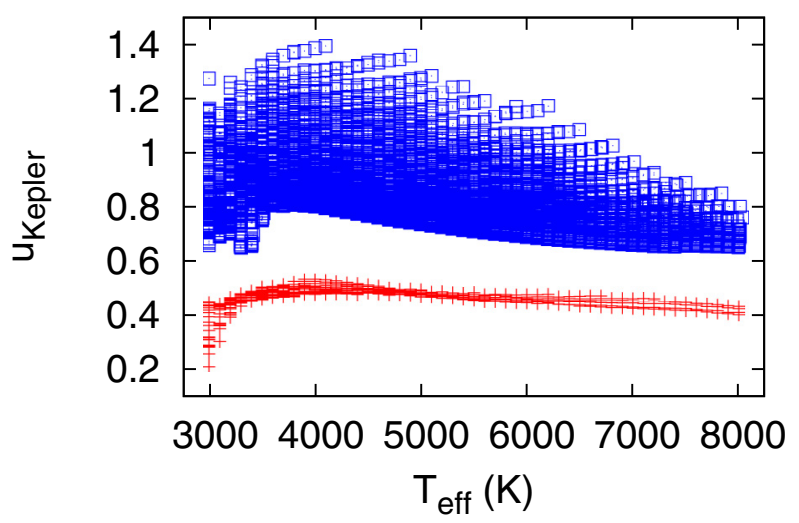

Fig. 2. Limb-darkening coefficient $u$, used in Eq. (1), applied to the Kepler photometric band. Red crosses are the plane-parallel model stellar atmospheres, and the blue squares are the spherical models.

less well than for the plane-parallel models because of this nonlinearity. For the model shown in Fig. 1, limb-darkening laws predict intensities that vary by $\Delta \equiv I_{\text {model }}-I_{\text {law }}=0.15$ for the spherical model while $\Delta<0.04$ for the plane-parallel model. Although limb-darkening laws fit the intensities of plane-parallel model atmospheres better than spherically symmetric models, the spherical models are more physically realistic, making them the more appropriate choice to use in modeling observations. We explore the uncertainty of the limb-darkening fits later.

We present in Figs. 2-5 the coefficients derived by leastsquares fitting for the limb-darkening laws given by Eqs. (1)-(6) respectively for the Kepler photometric band as a function of effective temperature for both plane-parallel and spherically symmetric model stellar atmospheres. It is clear that more realistic spherically symmetric model stellar atmospheres predict limbdarkening coefficients that vary much more as a function of effective temperature than those for plane-parallel models. For the simplest case of the linear limb-darkening law, the $u$-coefficient determined from plane-parallel models in the Kepler-band vary from $u=0.2$ to 0.5 , whereas spherical models with the same effective temperatures and gravities vary from $u=0.6$ to 1.4 . The coefficients predicted for almost all limb-darkening laws examined here show the same behavior as the limb-darkening coefficients predicted for a flux-conserving linear+square-root law (Neilson \& Lester 2011, 2012). This uniform dependence of the coefficients on $T_{\text {eff }}$ is surprising and suggests all of these laws carry essentially the same information regarding the moments of the intensity and the atmospheric extension about the stellar atmosphere in question. The one exception is the Claret (2000a) four-parameter limb-darkening law, for which the coefficients appear to vary much more as a function of effective temperature.

To explore the interdependence of the coefficients, we plot in Fig. 6 the Kepler-band $b$-coefficient from the quadratic law as a function of the $a$-coefficient. This plot is typical of all the twoparameter limb-darkening laws considered in this work as well as the limb-darkening law employed by Neilson \& Lester (2011, 2012), including the apparent hook in the correlation between coefficients. Figure 6 also plots the values of $f_{2}+f_{4}$ as a function of $f_{1}+f_{3}$ for the four-parameter law, again for the Kepler photometric band. The correlation for both plane-parallel and spherical models is readily apparent. A best-fit linear relation to the coefficients for spherical models is

$f_{2, \text { Kepler }}+f_{4, \text { Kepler }}=-0.989\left(f_{1, \text { Kepler }}+f_{3, \text { Kepler }}\right)+1.051$.

The correlation is different for plane-parallel models for which the slope is -0.978 and the intercept is 0.493 .

These correlations are caused by the limb-darkening coefficients being linear combinations of various angular moments of the intensity. For instance, in plane-parallel model atmospheres the moments $J \equiv \int I(\mu) \mathrm{d} \mu$ and $K \equiv \int I(\mu) \mu^{2} \mathrm{~d} \mu$ are related such that $J=3 K$ (Mihalas 1978). In spherical symmetry, this ratio varies, causing the moments of the intensity to differ in spherical symmetry from those predicted moments for plane-parallel model stellar atmospheres. This difference in geometry is reflected in the difference between the zero-points of the relation Eq. (7) for spherical models and that for plane-parallel models. One can potentially use this difference to test observations and test model geometry. 

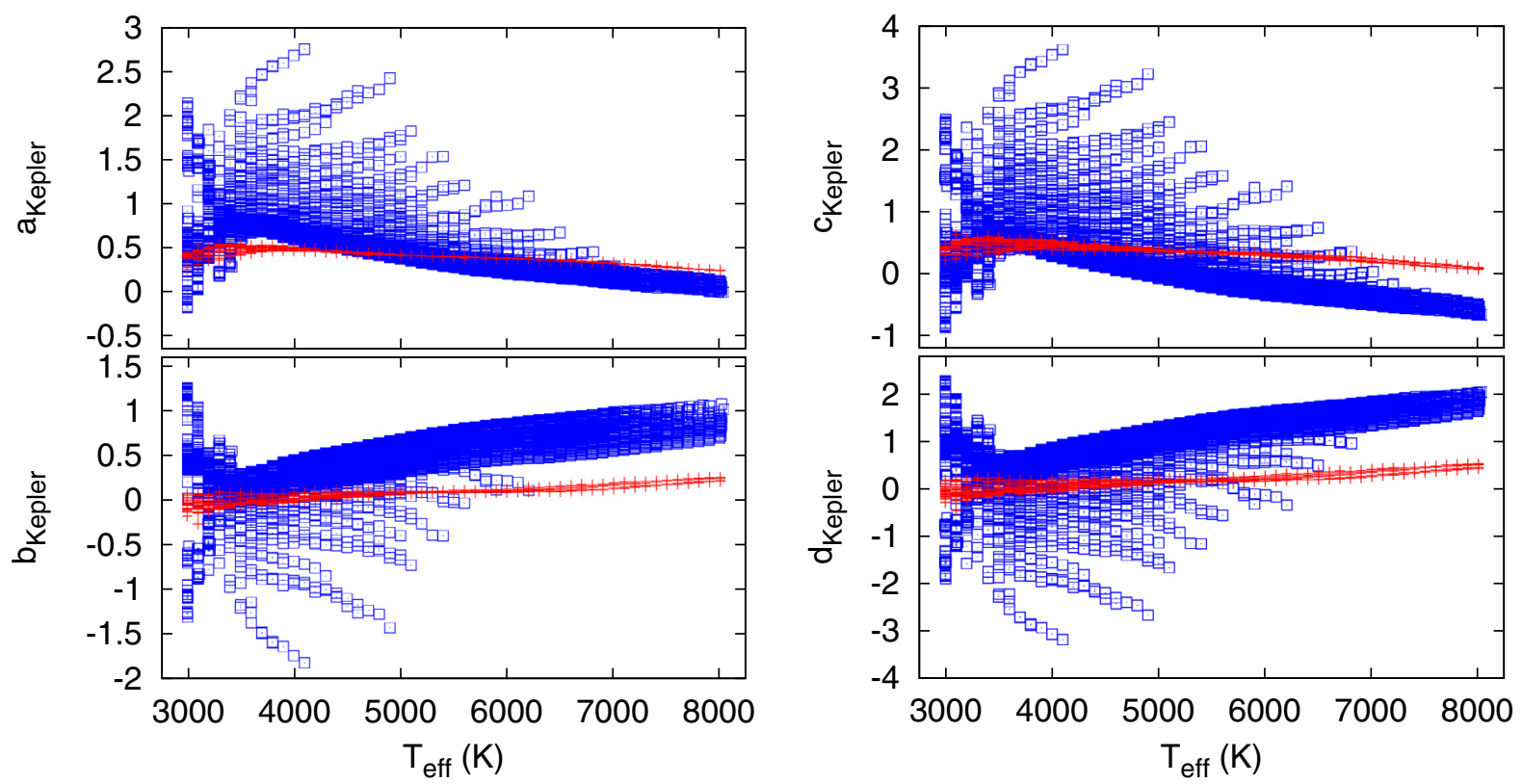

Fig. 3. Limb-darkening coefficients $a$ and $b$ used in Eq. (2) (left panel), and the coefficients $c$ and $d$ used in Eq. (3) (right panel), all applied to the Kepler photometric band. The symbols have the same meanings as in Fig. 2.
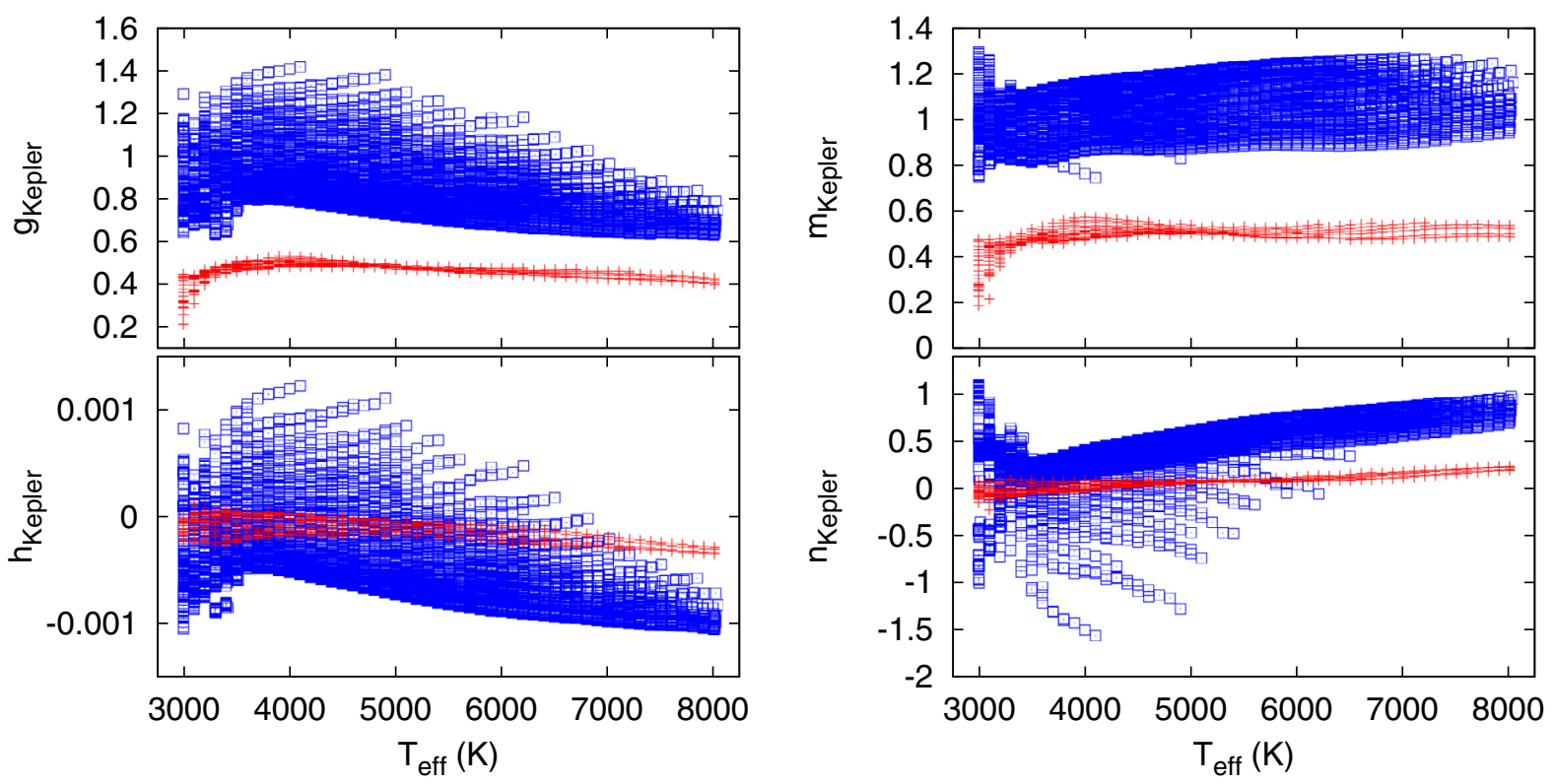

Fig. 4. Limb-darkening coefficients $g$ and $h$ used in Eq. (5) (left panel), and the coefficients $m$ and $n$ used in Eq. (6) (right panel), all applied to the Kepler photometric band. The symbols have the same meanings as in Fig. 2.

\subsection{Error analysis}

Various limb-darkening laws, such as those given in Eqs. (1)-(6), are fit to the surface intensities computed with model stellar atmospheres, and it is important to understand how well these laws represent the actual intensities. For instance, Diaz-Cordoves et al. (1995) argued that a square-root law fit intensity profiles for hotter stars $\left(T_{\text {eff }}>9000 \mathrm{~K}\right)$ better than a quadratic law, whereas no limb-darkening law is preferred for cooler stars. We compute the relative error of the limb-darkening fit, $\Delta$, using the relation

$\Delta_{\lambda} \equiv \sqrt{\frac{\sum\left[I_{\text {model }}(\mu)-I_{\text {fit }}(\mu)\right]^{2}}{\sum\left[I_{\text {fit }}(\mu)\right]^{2}}}$, which quantifies the deviation of the best-fit limb-darkening law from the surface intensities of the model atmosphere. We compute the relative error for each bandpass as a function of the fundamental stellar parameters for both plane-parallel and spherical geometries, and show in Fig. 7 the relative errors as a function of effective temperature for fits in the Kepler-band. The relative error of the fits for spherical models is greater than the error for plane-parallel fits for all the limb-darkening laws. The errors are similar only for $T_{\text {eff }} \sim 3500 \mathrm{~K}$, where the spherical model atmospheres predict intensity profiles that are closest to being linear, with the error of the linear limb-darkening law approaching a minimum value. This result appears to suggest that these limb-darkening laws are inappropriate for fitting light curves and interferometric observations, but this is not true. 
H. R. Neilson and J. B. Lester: Limb darkening in red giant stars
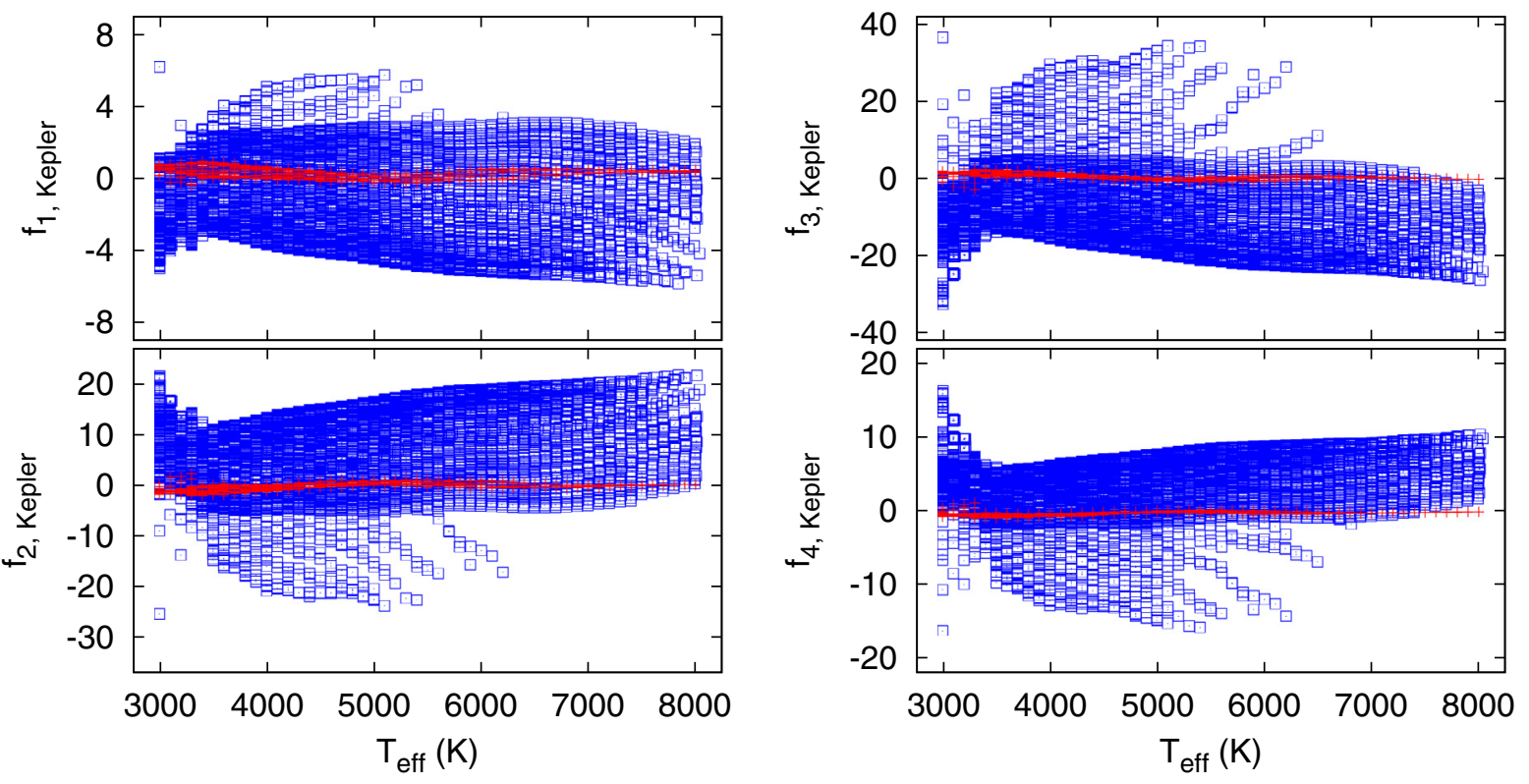

Fig. 5. Limb-darkening coefficients $f_{1}, f_{2}, f_{3}$ and $f_{4}$ used in the Claret (2000a) four-parameter law, Eq. (4), applied to the Kepler photometric band. The symbols are the same as in Fig. 2.
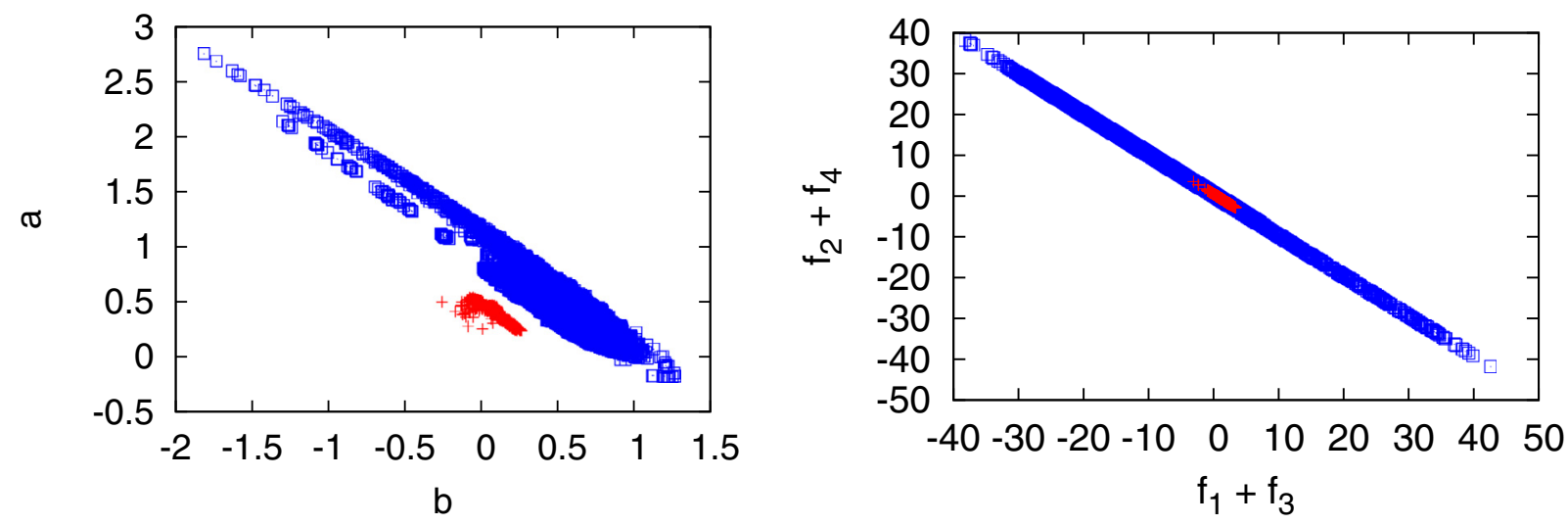

Fig. 6. Correlation between the Kepler-band limb-darkening coefficients for the quadratic law (left) and for the four-parameter law, $f_{2}+f_{4}$ as a function of $f_{1}+f_{3}(r i g h t)$. Red crosses represent coefficients computed from plane-parallel models and blue squares spherical models.

There are a number of issues with how the relative error is computed and what the error tells us, such as how the limbdarkening laws are defined, how they are fit to the surface intensities and the effect of sampling.

- Defining limb-darkening laws: The intensity profiles computed using the plane-parallel and spherical model atmospheres employed in this work are normalized with respect to the central intensity so that $I(\mu=1) \equiv 1$. Furthermore, all limb-darkening laws, except the exponential law, are defined so that the $I(\mu=1) \equiv 1$, regardless of the values of the bestfit coefficients. As a result, every fit to an intensity profile is anchored to the center of the stellar disk before representing the remainder of the intensity profile. This definition alone results in a perfect fit to the center of the stellar disk and a deteriorating fit as $\mu \rightarrow 0$ as the intensity profile deviates from the assumed structure of a particular limb-darkening law.

- Fitting limb-darkening laws: Limb-darkening laws are typically fit to intensity profiles using a least-square algorithm. Neilson \& Lester (2011) showed that the best-fit coefficients for a given law are functions of weighted integrals of the intensity profile. For example, the linear limb-darkening coefficient from Eq. (1) is a function of the mean intensity, $J$, and the stellar flux, $\mathcal{H} \equiv \int I(\mu) \mu \mathrm{d} \mu$, and both of these quantities are more sensitive to the central intensity than to the much smaller intensity near the limb. As with the definition of the limb-darkening laws, using a least square fitting algorithm fits the central part of the intensity structure better. Similarly, one might fit limb-darkening coefficients by enforcing flux conservation, but because the flux is the $\mu$-weighted integral of the intensity, any flux-conserving fit is constrained weakly by the intensity at the stellar limb relative to the intensity near the center of the stellar disk.

- Sampling issues: Sampling is the most important of the three issues affecting the computed error of the fit of the intensity profile. For instance, Wade \& Rucinski (1985) and Heyrovský (2007) noted that fitting an intensity profile that is uniformly sampled in $\mu$ has a larger error than fitting the same profile that is uniformly sampled in $r=$ $\sin \theta=\sin \left(\cos ^{-1} \mu\right)$. Uniform $r$-spacing emphasizes the intensity profile near the center of the disk while a uniform $\mu$-spacing emphasizes the limb. Adopting any of the limbdarkening laws presented here, that law will fit the central 

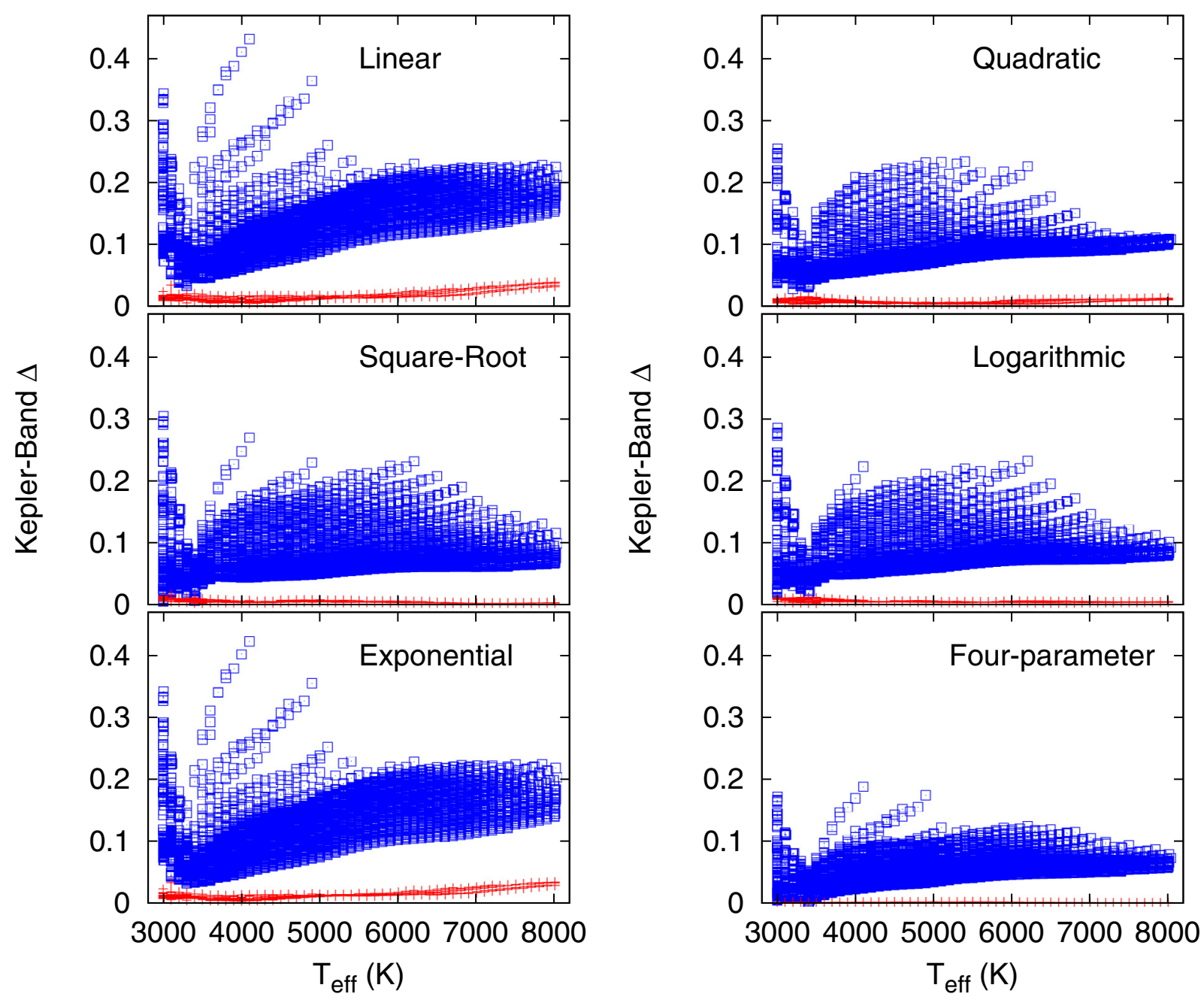

Fig. 7. Error of the best-fit limb-darkening relation, defined by Eq. (8), for every model atmosphere (red crosses represent plane-parallel models, blue squares spherical models) for each of the six limb-darkening laws at Kepler-band wavelengths.

part of the stellar surface more precisely than the limb because of the normalization at the center of the disk. If, in addition, the surface intensity is sampled uniformly in $r$, that will give added weight to the central region. These two factor combine to make the computed error of the fit smaller. Similarly, Howarth (2011b) found that limb-darkening coefficients derived from planetary transits with large impact factors do not agree with model stellar atmosphere predictions. This is because the planet passes across only the limb of the star and not the center, therefore probing only part of the intensity profiles. Claret $(2008,2009)$ also found disagreement between theoretical limb-darkening coefficients and empirical coefficients measured from eclipsing binary light curves and comparisons to the planetary system HD 209458. Limbdarkening coefficients from stellar atmosphere models fit the whole profile yielding different results.

The combination of these three factors lead to calculated errors that are relative and not an absolute measure of the quality of the fit. In this work, differences in the error between fits to planeparallel and spherically symmetric model stellar atmosphere intensity profiles computed with the same properties are due solely to differences in the intensity profile near the limb where the spherical models provide more realistic predictions. Therefore, the error analysis suggests that the various limb-darkening laws lack the necessary complexity to precisely fit intensity profiles from spherical models. The only exception is the Claret (2000a) four-parameter law, which fits the laws best, but appears to have unique properties.

\section{Gravity darkening coefficients}

Rapid rotation distorts the shape of a star, making it aspheric, with flattened poles and a bulged equator. As shown first by von Zeipel (1924), the gravity and effective temperature vary in a coordinated way across the stellar surface such that at any point the effective temperature is proportional to the effective gravity, $T_{\mathrm{eff}} \sim g_{\mathrm{eff}}^{\beta_{1} / 4}$, where $\beta_{1}=1$ for radiative stars. However, this value of $\beta_{1}$ is valid only for bolometric radiation, and Kopal (1959) later derived monochromatic gravity-darkening corrections, $y(\lambda)$. Claret (2000a), Claret \& Hauschildt (2003) and Claret \& Bloemen (2011) have computed waveband-dependent gravity-darkening corrections as a a function of the central intensity of the star, as well as the gravity, effective temperature and the variable, $\beta_{1}$ from plane-parallel models. Bloemen et al. (2011) derived

$y(\lambda)=\left(\frac{\partial \ln I(\lambda)}{\partial \ln g}\right)_{T_{\mathrm{eff}}}+\left(\frac{\mathrm{d} \ln T_{\mathrm{eff}}}{\mathrm{d} \ln g}\right)\left(\frac{\partial \ln I(\lambda)}{\partial \ln T_{\mathrm{eff}}}\right)_{g}$,

and noted that $\left(\mathrm{d} \ln T_{\mathrm{eff}} / \mathrm{d} \ln g\right)=\beta_{1} / 4$. The variable $\beta_{1}$ is a function of effective temperature, but for the purpose of this analysis 
H. R. Neilson and J. B. Lester: Limb darkening in red giant stars
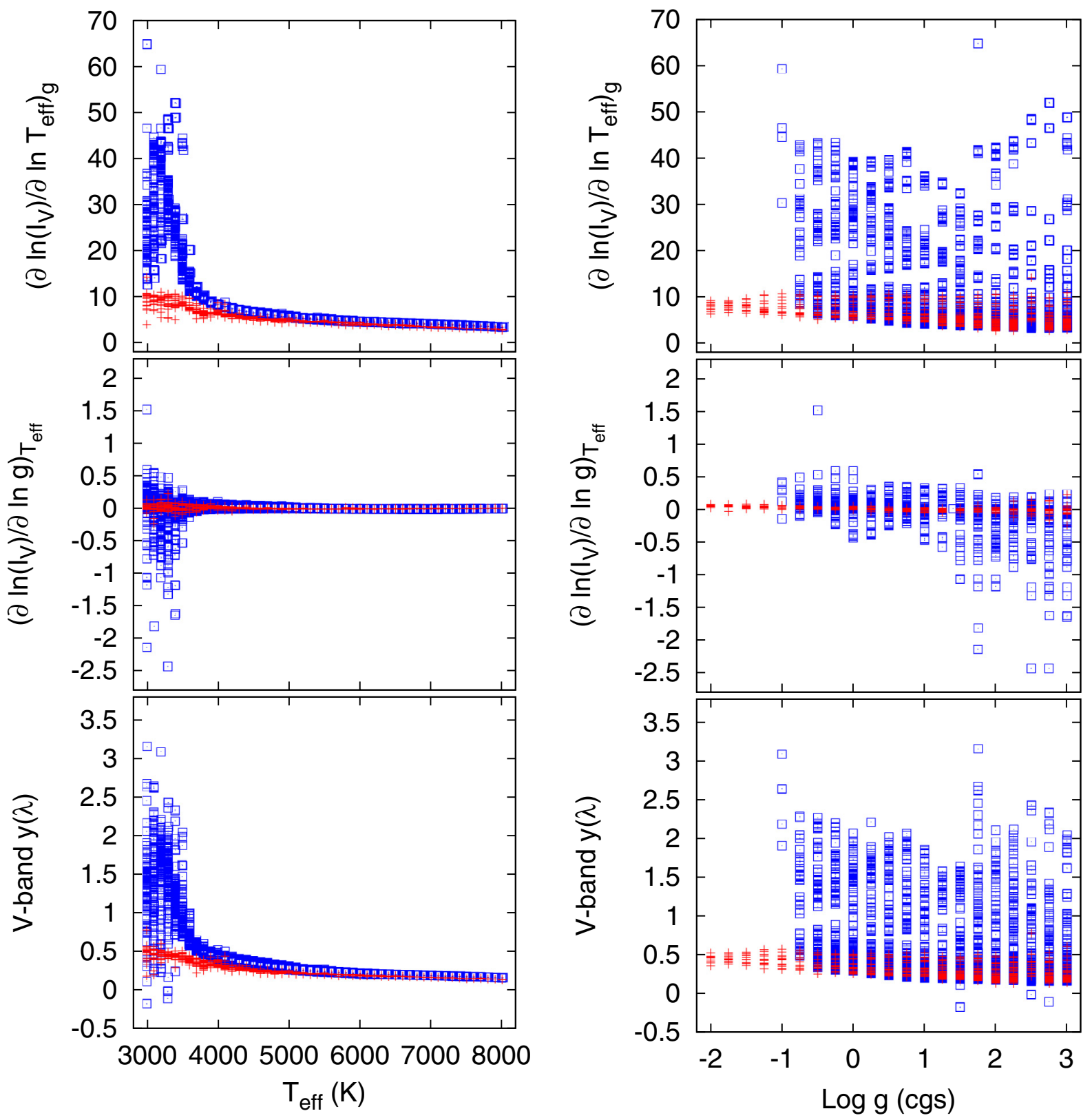

Fig. 8. $V$-band central intensity derivatives and gravity-darkening coefficients as function of effective temperature (left) and gravity (right) computed from plane-parallel (red crosses) and spherically symmetric (blue squares) model stellar atmospheres.

we assume $\beta_{1}=0.2$ for $T_{\text {eff }}<7500 \mathrm{~K}$ and $\beta_{1}=1$ for hotter stars. However, the value of $\beta_{1}$ based on von Zeipel's theorem is not strictly valid for radiative or convective stellar envelopes (Claret 2000b, 2012; Espinosa Lara \& Rieutord 2011).

In Fig. 8, we plot the $V$-band values of each intensity derivative for each model stellar atmosphere in Eq. (9), as well as $y(\lambda)$ computed for the assumed values of $\beta_{1}$. We find that planeparallel and spherically symmetric model stellar atmospheres predict similar gravity-darkening coefficients for $T_{\text {eff }}>4000 \mathrm{~K}$, but there are significant differences for cooler stars. We interpret these differences for the cooler stars as consequences of both surface convection and the shift from the negative hydrogen ion to titanium oxide as the dominant opacity source. Both planeparallel and spherical model intensities show greater variation at these cool effective temperatures, but the intensity profiles of spherically symmetric model atmospheres vary more than that of plane-parallel model atmospheres.
While the most significant differences between spherical and planar model predictions of gravity-darkening coefficients are at lower temperatures, the gravity-darkening coefficients computed from spherically symmetric models are greater than those of plane-parallel models for every effective temperature. For example, a spherically symmetric model with $T_{\text {eff }}=8000 \mathrm{~K}$ has a $V$-band gravity-darkening coefficient of $y_{V} \simeq 0.165$ while the plane-parallel model with the same effective temperature has $y_{V} \simeq 0.14$. The difference is small but systematic.

\section{Angular diameter corrections}

Interferometric observations measure the angular diameter of a star along with its limb-darkening profile, but, unfortunately, the measured angular diameter and limb-darkening profiles are not independent quantities. This is especially true when the measured visibilities do not probe the second lobe. 

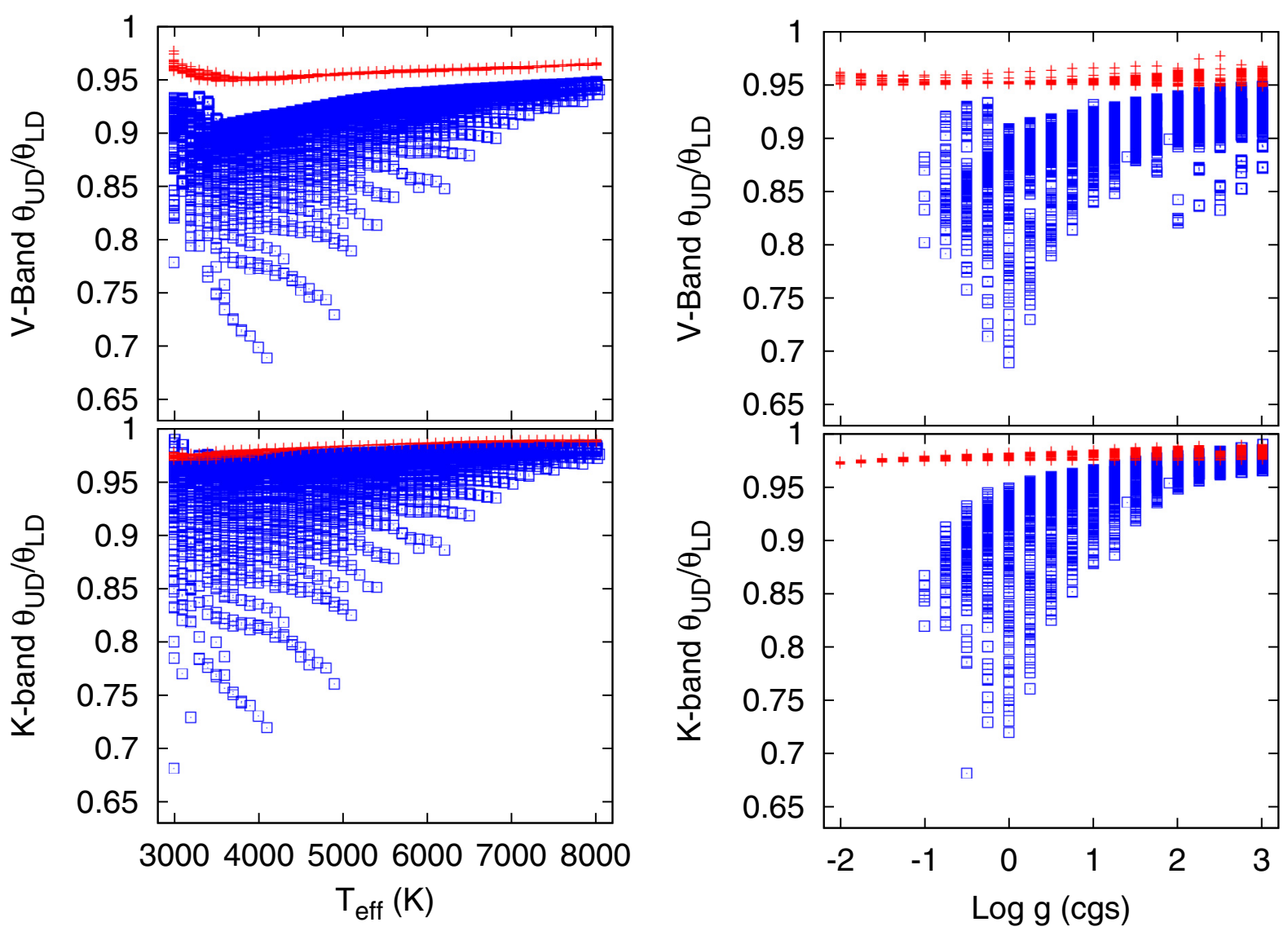

Fig. 9. Interferometric angular diameter correction computed in $V$-band (top) and $K$-band as functions of effective temperature (left) and gravity (right). Corrections computed from plane-parallel model atmospheres are denoted with red x's and spherically symmetric models blue squares.

Davis et al. (2000) measured stellar angular diameters from interferometric observations by assuming that the stellar intensity profile is uniform, i.e. the intensity at any point on a stellar disk is equal to the central intensity. In that case, the uniform-disk angular diameter can be directly fit to the observed visibilities and then converted to a limb-darkened angular diameter using model stellar atmospheres. Davis et al. (2000) computed corrections using plane-parallel ATLAS models (Kurucz 1993) and found $k \equiv \theta_{\mathrm{UD}} / \theta_{\mathrm{LD}}=0.91$ to 0.98 in the wavelength range $\lambda=400-800 \mathrm{~nm}$. These limb-darkening corrections have been applied to observations of Cepheids (Gallenne et al. 2012) and Sirius (Davis et al. 2011) for example.

We compute angular diameter corrections using the recipe described by Marengo et al. (2004), where we assume a limbdarkened angular diameter of $\theta_{\mathrm{LD}}=1$ mas to compute interferometric visibilities from a model atmosphere intensity profile. That synthetic visibility is then fit by a uniform-disk angular diameter. The best-fit uniform-disk angular diameter is then equivalent to the theoretical angular diameter correction. We compute angular diameter corrections for the Johnson-Cousins BVRIHK wavebands and show the corrections for the $V$ - and $K$-bands in Fig. 9 as a function of effective temperature for plane-parallel and spherically symmetric models. Corrections from spherical models clearly differ from corrections from plane-parallel model atmospheres. Intensity profiles from plane-parallel model stellar atmospheres predict corrections in the narrow range from $k=0.97-0.99$ in $V$-band and approaches unity for longer wavelengths. We show in Fig. 9 the $V$ - and $K$-band angular diameter corrections as function of effective temperature and gravity for plane-parallel and spherically symmetric model stellar atmospheres.
Fits to spherically-symmetric model atmospheres suggest significantly different angular diameter corrections as functions of both effective temperature and gravity. The $V$-band corrections from spherical models, denoted $k_{\mathrm{s}}$, range from $k_{\mathrm{s}}=0.67$ to 0.95 , with no overlap with the plane-parallel model predictions. The $K$-band corrections show similar behaviors except that spherical and planar corrections overlap somewhat. These results suggest that using plane-parallel model atmosphere corrections systematically underestimates the stellar angular diameter. For instance, Mozurkewich et al. (2003) presented uniform-disk angular diameters for a sample of 85 stars, along with limbdarkened angular diameters corrected using limb-darkening coefficients from Claret et al. (1995) and Diaz-Cordoves et al. (1995). Their angular diameter corrections vary from $k=0.89$ to $\approx 1$, consistent with the values found here for plane-parallel model atmospheres.

Of particular interest are the results of Mozurkewich et al. (2003) for $\alpha$ Persei (F5 Ib), for which they measured $T_{\text {eff }}=$ $6750 \mathrm{~K}$, and for $\epsilon$ Geminorum (G8 Ib), which was measured to have $T_{\text {eff }}=4485 \mathrm{~K}$. Mozurkewich et al. (2003) measured the uniform-disk angular diameters at $550 \mathrm{~nm}$ to be $2.986 \pm 0.042$ for $\alpha$ Per and $4.467 \pm 0.115$ mas for $\epsilon$ Gem. Using these they computed limb-darkened angular diameters of $3.188 \pm 0.035$ and $4.703 \pm 0.047$ mas, respectively. Our spherically-symmetric models with $\log g=1.5$ and $M=10 M_{\odot}$ yield $V$-band angulardiameter corrections of 0.929 for $\alpha$ Per and 0.916 for $\epsilon$ Gem. Applying these to the uniform disk measurements gives larger limb-darkened angular diameters: $\theta_{\mathrm{LD}}=3.214$ mas for $\alpha$ Per and $\theta_{\mathrm{LD}}=4.877$ mas for $\epsilon \mathrm{Gem}$. The spherical correction for $\alpha$ Per yields a value for $\theta_{\mathrm{LD}}$ that is marginally consistent with the angular diameter found using plane-parallel correction, 


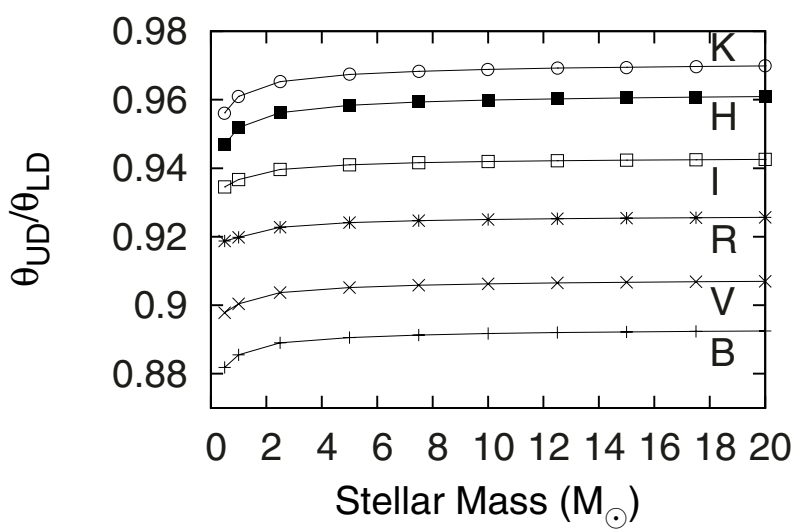

Fig. 10. Interferometric angular diameter corrections as a function of waveband and stellar mass for spherically symmetric model stellar atmospheres with $\log g=2$ and $T_{\text {eff }}=3500 \mathrm{~K}$.

whereas the limb-darkened angular diameter of $\epsilon$ Gem measured by Mozurkewich et al. (2003) is almost 4\% smaller than what would be predicted by applying spherical model corrections. This difference may appear to be small but this underestimate is systematic.

As a test, we check how the angular diameter corrections vary as function of stellar mass. Because models with low effective temperature but relatively high gravity appear to predict the smallest corrections, we hold $T_{\text {eff }}=3500 \mathrm{~K}$ and $\log g=2$. The angular diameter corrections are shown in Fig. 10 as a function of stellar mass for the six Johnson-Cousins wavebands considered in this work. The figure suggests that the corrections are insensitive to the mass of the stellar model except for low-mass $\left(M \leq 1 M_{\odot}\right)$ models. This is reassuring and suggests that when applying these corrections, one can ignore the stellar mass. The difference between limb-darkening profiles and angular diameter corrections is small and consistent with previous results by Lester et al. (2013).

\section{Summary}

In this work, we present model atmosphere intensity profiles for the BVRIHK, CoRot and Kepler passbands from both planeparallel and spherically symmetric geometries based on models computed by Neilson \& Lester $(2011,2012)$. We fit a number of limb-darkening laws to these intensity profiles, as well as compute gravity-darkening coefficients and angular diameter corrections for interferometry. We test how these fits vary as a function of model atmosphere geometry and compile tables of limb-darkening coefficients, gravity-darkening coefficients and angular diameter corrections that can be applied to observations.

We consider six limb-darkening laws in this work: linear, quadratic, square-root, four-parameter, exponential and logarithmic. These laws fit the intensity profiles from plane-parallel model atmospheres well, but not the intensity profiles of the spherical models based on computed relative errors. The one exception is the Claret (2000a) four-parameter law, for which the difference between the spherical model intensity profiles and the predictions of the fitting law is small enough to still be applicable to observations, although the law still fits the spherical profiles more poorly than the plane-parallel intensities.

While those predicted errors are useful for comparing fits to planar and spherical model intensity profiles, they are not ideal for studies of actual limb darkening. Best-fit limb-darkening coefficients depend on the definition of the laws, all of which anchor the fit to $I(\mu=1)=1$, making the fit sensitive to the sampling of the intensity profile as well as to the method for fitting the data. Because intensity profiles for spherical models are more complex, the fitting error is greater than the error for simpler plane-parallel model intensity profiles. However, spherically symmetric model atmospheres are a more realistic representation of actual stellar atmospheres, meaning they are better suited for limb darkening studies.

Fits to the four-parameter limb-darkening law also show correlations between the limb-darkening coefficients; we find that the linear combination of the four coefficients are approximately constant, with that constant being a function of the atmosphere's geometry. This result suggests that the linear combination of the observed coefficients for the four-parameter law provides a simple test of whether the observations are probing the edge of the stellar disk, i.e. sphericity.

We also predict wavelength-dependent gravity-darkening coefficients based on the Claret \& Bloemen (2011) prescription. Unlike the limb-darkening coefficients, the gravity-darkening coefficients are less dependent on model atmosphere geometry. This is because the gravity-darkening coefficients depend on the change of the central intensity with respect to effective temperature and gravity, hence the difference between atmospheres for the same geometry. Gravity-darkening is also a function of the central intensity, which is insensitive to model geometry. The spherically symmetric gravity-darkening coefficients are similar to plane-parallel coefficients for $T_{\text {eff }}>5000 \mathrm{~K}$ and begin to diverge for cooler stellar atmosphere models. Only at the coolest effective temperatures, $3000 \mathrm{~K} \leq T_{\text {eff }} \leq 4000 \mathrm{~K}$, is the geometry of the model atmosphere important, with the spherically symmetric coefficients being approximately an orderof-magnitude greater than those predicted from plane-parallel model atmospheres.

Unlike the gravity darkening coefficients, the interferometric angular-diameter corrections do depend on geometry. For planeparallel model atmospheres the angular-diameter corrections vary from about $0.95-1$, whereas the corrections for spherically symmetric model atmospheres vary from $0.67-1$. Previous analyses had assumed that corrections from plane-parallel models are applicable to all stars, but this is not true. At low gravity, $\log g<3$, spherically symmetric corrections deviate significantly from plane-parallel model predictions. The difference between spherical and plane-parallel models is a function of both gravity and effective temperature and also appears to vary as a function of stellar mass.

The angular-diameter corrections, limb-darkening and gravity-darkening coefficients are publicly available as online tables. Each table has the format $T_{\text {eff }}(\mathrm{K}), \log g$, and $M\left(M_{\odot}\right)$ and then the appropriate variables for each waveband, such as linear limb-darkening coefficients. Tables of gravity-darkening coefficients also contain values of the intensity derivatives with respect to gravity and effective temperature. For plane-parallel models, only $T_{\text {eff }}$ and $\log g$ are given in the tables. We list the properties of these tables in Table 1, that are archived in electronic form at the CDS. Model atmosphere intensity profiles are also archived at the CDS

Techniques such as optical interferometry, microlensing observations, planetary transit and eclipsing binary observations are continuously improving the measurements of stellar limb darkening needed to test model stellar atmospheres and the physics assumed in their calculation. At lower gravities, these observations require the more physically realistic spherically symmetric models to constrain stellar properties. 
Table 1. Summary of limb-darkening coefficient, gravity-darkening coefficient and interferometric angular diameter correction tables found online.

\begin{tabular}{lll}
\hline \hline Name & Geometry & Type \\
\hline Table2 & Spherical & Linear limb darkening Eq. (1) \\
Table3 & Spherical & Quadratic limb darkening Eq. (2) \\
Table4 & Spherical & Square root limb darkening Eq. (3) \\
Table5 & Spherical & Four-parameter limb darkening Eq. (4) \\
Table6 & Spherical & Exponential limb darkening Eq. (5) \\
Table7 & Spherical & Logarithmic limb darkening Eq. (6) \\
Table8 & Planar & Linear limb darkening Eq. (1) \\
Table9 & Planar & Quadratic limb darkening Eq. (2) \\
Table10 & Planar & Square root limb darkening Eq. (3) \\
Table11 & Planar & Four-parameter limb darkening Eq. (4) \\
Table12 & Planar & Exponential limb darkening Eq. (5) \\
Table13 & Planar & Logarithmic limb darkening Eq. (6) \\
Table14 & Spherical & Gravity darkening \\
Table15 & Planar & Gravity darkening \\
Table16 & Spherical & Angular diameter corrections \\
Table17 & Planar & Angular diameter corrections \\
\hline
\end{tabular}

Notes. Tables listed here can be retrieved electronically from the CDS.

The predicted limb-darkening coefficients, gravity-darkening coefficients and angular diameter corrections from spherically symmetric SATLAS models are new tools that for aiding analyses of these observations.

Acknowledgements. The authors acknowledge support from a research grant from the Natural Sciences and Engineering Research Council of Canada, the Alexander von Humboldt Foundation and National Science Foundation (AST-0807664).

\section{References}

Al-Naimiy, H. M. 1978, Ap\&SS, 53, 181

An, J. H., Albrow, M. D., Beaulieu, J.-P., et al. 2002, ApJ, 572, 521

Aufdenberg, J. P., Ludwig, H.-G., \& Kervella, P. 2005, ApJ, 633, 424

Auvergne, M., Bodin, P., Boisnard, L., et al. 2009, A\&A, 506, 411

Avrett, E. H., \& Krook, M. 1963, ApJ, 137, 874

Bessell, M. S. 2005, ARA\&A, 43, 293

Bloemen, S., Marsh, T. R., Østensen, R. H., et al. 2011, MNRAS, 410, 1787

Catala, C., Arentoft, T., Fridlund, M., et al. 2010, in Pathways Towards Habitable Planets, eds. V. Coudé Du Foresto, D. M. Gelino, \& I. Ribas, ASP Conf. Ser., 430, 260

Claret, A. 2000a, A\&A, 363, 1081

Claret, A. 2000b, A\&A, 359, 289

Claret, A. 2008, A\&A, 482, 259

Claret, A. 2009, A\&A, 506, 1335
Claret, A. 2012, A\&A, 538, A3

Claret, A., \& Bloemen, S. 2011, A\&A, 529, A75

Claret, A., \& Hauschildt, P. H. 2003, A\&A, 412, 241

Claret, A., Diaz-Cordoves, J., \& Gimenez, A. 1995, A\&AS, 114, 247

Claret, A., Hauschildt, P. H., \& Witte, S. 2012, A\&A, 546, A14

Claret, A., Hauschildt, P. H., \& Witte, S. 2013, A\&A, 552, A16

Croll, B., Albert, L., Jayawardhana, R., et al. 2011, ApJ, 736, 78

Davis, J., Tango, W. J., \& Booth, A. J. 2000, MNRAS, 318, 387

Davis, J., Ireland, M. J., North, J. R., et al. 2011, PASA, 28, 58

Diaz-Cordoves, J., Claret, A., \& Gimenez, A. 1995, A\&AS, 110, 329

Eaton, J. A., Henry, G. W., \& Odell, A. P. 2008, ApJ, 679, 1490

Espinosa Lara, F., \& Rieutord, M. 2011, A\&A, 533, A43

Feautrier, P. 1964, Comptes Rendus Academie des Sciences (série non spécifiée), 258, 3189

Fields, D. L., Albrow, M. D., An, J., et al. 2003, ApJ, 596, 1305

Fouqué, P., Heyrovský, D., Dong, S., et al. 2010, A\&A, 518, A51

Gallenne, A., Kervella, P., Mérand, A., et al. 2012, A\&A, 541, A87

Gustafsson, B., Edvardsson, B., Eriksson, K., et al. 2008, A\&A, 486, 951

Hanbury Brown, R., Davis, J., Lake, R. J. W., \& Thompson, R. J. 1974, MNRAS, 167,475

Haubois, X., Perrin, G., Lacour, S., et al. 2009, A\&A, 508, 923

Hauschildt, P. H., Allard, F., Ferguson, J., Baron, E., \& Alexander, D. R. 1999, ApJ, 525, 871

Heyrovský, D. 2007, ApJ, 656, 483

Howarth, I. D. 2011a, MNRAS, 413, 1515

Howarth, I. D. 2011b, MNRAS, 418, 1165

Howell, S. B., Rowe, J. F., Bryson, S. T., et al. 2012, ApJ, 746, 123

Johnson, H. L., \& Morgan, W. W. 1953, ApJ, 117, 313

Koch, D. G., Borucki, W., Dunham, E., et al. 2004, in SPIE Conf. Ser. 5487, ed. J. C. Mather, 1491

Kopal, Z. 1959, Close binary systems (London: Chapman \& Hall)

Kurucz, R. L. 1979, ApJS, 40, 1

Kurucz, R. L. 1993, in IAU Colloq. 138: Peculiar versus Normal Phenomena in A-type and Related Stars, eds. M. M. Dworetsky, F. Castelli, \& R. Faraggiana, ASP Conf. Ser., 44, 87

Lester, J. B., \& Neilson, H. R. 2008, A\&A, 491, 633

Lester, J. B., Dinshaw, R., \& Neilson, H. R. 2013, PASP, 125, 335

Marengo, M., Karovska, M., Sasselov, D. D., \& Sanchez, M. 2004, ApJ, 603, 285

Mihalas, D. 1978, Stellar atmospheres, 2nd edition (San Francisco: W. H. Freeman and Co.)

Mozurkewich, D., Armstrong, J. T., Hindsley, R. B., et al. 2003, AJ, 126, 2502

Neilson, H. R., \& Lester, J. B. 2008, A\&A, 490, 807

Neilson, H. R., \& Lester, J. B. 2011, A\&A, 530, A65

Neilson, H. R., \& Lester, J. B. 2012, A\&A, 544, A117

Rybicki, G. B. 1971, J. Quant. Spec. Radiat. Transf., 11, 589

Schwarzschild, K. 1906, Nachrichten von der Königlichen Gesellschaft der Wissenschaften zu Göttingen, Mathematisch-physikalische Klasse, 41

Sing, D. K. 2010, A\&A, 510, A21

van Belle, G. T., Ciardi, D. R., Thompson, R. R., Akeson, R. L., \& Lada, E. A. 2001, ApJ, 559, 1155

van Hamme, W. 1993, AJ, 106, 2096

von Zeipel, H. 1924, MNRAS, 84, 665

Wade, R. A., \& Rucinski, S. M. 1985, A\&AS, 60, 471

Wittkowski, M., Aufdenberg, J. P., \& Kervella, P. 2004, A\&A, 413, 711

Zub, M., Cassan, A., Heyrovský, D., et al. 2011, A\&A, 525, A15 